\title{
Cécile OTTOGALLI-MAZZACAVALLO, Femmes et
} alpinisme. 1874-1919: un genre de compromis, Paris, L'Harmattan, 2006, 312 pages (Coll. « Espaces et Temps du sport »).

\section{Christine Mennesson}

\section{OpenEdition}

Journals

Édition électronique

URL : https://journals.openedition.org/clio/6983

DOI : $10.4000 /$ clio.6983

ISSN : 1777-5299

Éditeur

Belin

Édition imprimée

Date de publication : 1 novembre 2007

Pagination : 232-264

ISBN : 978-2-85816-940-5

ISSN : 1252-7017

\section{Référence électronique}

Christine Mennesson, «Cécile OTTOGALLI-MAZZACAVALLO, Femmes et alpinisme. 1874-1919: un genre de compromis, Paris, L'Harmattan, 2006, 312 pages (Coll. «Espaces et Temps du sport »). », Clio. Femmes, Genre, Histoire [En ligne], 26 | 2007, mis en ligne le 14 avril 2008, consulté le 24 avril 2022. URL : http://journals.openedition.org/clio/6983 ; DOI : https://doi.org/10.4000/clio.6983

Ce document a été généré automatiquement le 24 avril 2022

Tous droits réservés 


\title{
Cécile OTTOGALLI-MAZZACAVALLO, Femmes et alpinisme. 1874-1919: un genre de compromis, Paris, L'Harmattan, 2006, 312 pages (Coll. « Espaces et Temps du sport »).
}

\author{
Christine Mennesson
}

1 L'ouvrage de Cécile Ottogalli-Mazzacavallo aborde la question de la transformation des rapports sociaux de sexe et des définitions du genre au tournant du XXe siècle à partir des expériences originales des pionnières de la pratique de l'alpinisme entre 1874 et 1919. En repérant les étapes de l'évolution de l'alpinisme féminin, les modalités privilégiées par les différentes générations de femmes et les discours et représentations qui fondent leur pratique, l'auteure analyse les conditions sociales et les formes prises par un processus d'autonomisation relativement emblématique des transformations à l'œuvre dans la société française de l'époque. Cependant, si la féminisation très progressive des pratiques alpines s'inscrit dans une conjoncture spécifique, elle ne peut se comprendre sans tenir compte des origines sociales aisées des femmes concernées et de la position spécifique de l'alpinisme dans l'espace des pratiques physiques et sportives du moment. Ainsi, tout en donnant à voir des processus relativement génériques, l'auteure précise dans le même temps les différents éléments qui structurent de manière spécifique l'appropriation par les femmes de ces formes modernes d'aventure.

2 La première partie de l'ouvrage, organisé de manière thématique, porte sur la place des femmes dans l'activité - des pionnières aux adhérentes du Club Alpin Français (CAF), institution qui joue un rôle central dans la féminisation de l'alpinisme français. En effet, contrairement à la plupart des autres sociétés ou clubs sportifs qui refusent généralement d'intégrer des femmes, le CAF incite au développement d'une pratique alpine féminine, tout en contrôlant les modalités de pratique de ces dernières. Les 
vertus hygiéniques, morales et économiques de l'alpinisme se déclinent ainsi au féminin. De la promotion des pratiques touristiques ouvertes au plus grand nombre à l'accompagnement des maris et l'initiation des enfants, la présence de femmes «en bonne santé » et dotées d'une certaine capacité d'initiative sert plusieurs objectifs. L'intégration institutionnelle de la pratique des femmes respecte néanmoins la hiérarchie des sexes : «l'excursionnisme féminin » conjugue facilité et contemplation, laissant les prouesses physiques et les intérêts scientifiques à "l'excursionnisme cultivé » des hommes.

Dans la deuxième partie de l'ouvrage, l'auteure centre son analyse sur les pratiques et les représentations des femmes alpinistes. Malgré la permanence du processus de hiérarchisation entre hommes et femmes, les définitions du féminin et les formes de pratique de l'alpinisme qui lui sont associées évoluent. Le développement de l'alpinisme familial, les caravanes scolaires de jeunes filles, puis l'engagement de jeunes femmes émancipées constituent autant d'étapes vers une pratique alpine féminine qui se rapproche de la pratique des hommes et s'inscrit dans une démarche d'individuation. L'augmentation et la complexification de la pratique des femmes ne s'accomplissent néanmoins pas de manière linéaire et s'accompagnent par ailleurs d'une sportivisation de la pratique des hommes. En ce sens, si les femmes alpinistes imposent des usages du corps relativement novateurs, elles maintiennent néanmoins, par leur tenue vestimentaire ou la minoration de leurs exploits, la distance qui les sépare de leurs homologues masculins.

4 L'alpinisme féminin peut-il être considéré comme une forme de féminisme ? Cette question organise la troisième partie de l'ouvrage, centré sur les conditions sociales permettant l'émancipation des femmes et sur le rôle du CAF dans ce processus. Les exemples de Gabrielle Vallot et Mary Paillon dessinent tout d'abord les parcours atypiques de femmes contestant l'ordre social. L'auteure compare ensuite les positions adoptées par le CAF et celles d'institutions anglaises et suisses similaires. Le féminisme "modéré » du CAF apparaît bien comme une situation originale, qui permet aux pratiquantes de transformer en douceur les pratiques et les représentations des hommes, en acceptant des compromis toujours renouvelés entre le respect de la hiérarchie des sexes et leur volonté d'émancipation.

5 Cette histoire des femmes alpinistes au tournant du XXe siècle, basée sur des sources nombreuses et diversifiées, permet de mieux comprendre le rôle de la pratique sportive dans les processus de reproduction mais aussi de contestation de la domination masculine. Elle donne à voir les stratégies élaborées par ces « avant-gardistes cafistes » pour négocier l'évolution des rapports sociaux de sexe dans une institution et une conjoncture spécifiques. En ce sens, elle apporte des informations intéressantes, parfois présentées de manière descriptive et répétitive, sur l'histoire des femmes et les conditions de leur émancipation. On peut néanmoins s'étonner de la référence de l'auteure à un alpinisme permettant aux femmes de " découvrir leur vraie nature ». Le texte n'est ainsi pas toujours exempt d'un certain essentialisme et d'une vision homogénéisatrice de la condition des femmes au tournant du siècle. Si l'auteure s'inscrit dans la problématique de la construction sociale du genre, elle renvoie peu aux travaux qui fondent la réflexion sur les enjeux relatifs à la naturalisation du corps des femmes. 